\title{
Correction to: Enhancing SIEM Technology to Protect Critical Infrastructures
}

\author{
Luigi Coppolino, Salvatore D’Antonio, Valerio Formicola, \\ and Luigi Romano
}

\section{Correction to: \\ Chapter "Enhancing SIEM Technology to Protect Critical Infrastructures" in: B. Hämmerli, N. Kalstad Svendsen, and J. Lopez (Eds.): Critical Information Infrastructures \\ Security, LNCS 7722, \\ https://doi.org/10.1007/978-3-642-41485-5_2}

In the original version of this chapter, reference 9 "INSPIRE project" contained a link which was hacked and led to wrong content. Therefore, the link in reference 9 was omitted.

\footnotetext{
The updated version of this chapter can be found at https://doi.org/10.1007/978-3-642-41485-5_2

B. Hämmerli, N. Kalstad Svendsen, and J. Lopez (Eds.): CRITIS 2012, LNCS 7722, p. C1, 2013. 